\title{
DNA Damage Induced Cellular Senescence and It's PTEN Armed Exosomes - The Warriors Against Prostate Carcinoma Cells
}

Parimal Karmakar ( $\sim$ pkarmakar_28@yahoo.co.in )

Jadavpur University Faculty of Science https://orcid.org/0000-0002-9423-9906

Sougata Ghosh Chowdhury

Jadavpur University Faculty of Science

Rachayeeta Ray

Jadavpur University Faculty of Science

Debalina Bhattacharya

Maulana Azad College

Research Article

Keywords: Exosomes, Prostate carcinoma cells (PC3), PTEN, Senescence, DNA damage

Posted Date: October 12th, 2021

DOl: https://doi.org/10.21203/rs.3.rs-896768/v1

License: (c) (i) This work is licensed under a Creative Commons Attribution 4.0 International License.

Read Full License 


\section{Abstract}

Exosomes are one type of small extracellular vesicles having a size range of 30-150 $\mathrm{nm}$ and secreted by the endosomal compartment of most eukaryotic cells. It has been found that exosomes can serve as a communicating vehicle to transfer information among cells and thus can be associated with numerous physiological and pathological functions. In this study, we have isolated exosomes from two different human cancer cell lines. Isolated exosomes were characterized by scanning electron microscopy, DLS and by western blotting. It was observed that exosomes isolated from mock treated human lung epithelial carcinoma (A549) cells or Hela cells exerted growth arrest to the human prostate carcinoma (PC3) cells, but no growth arrest was observed in case of normal human lung fibroblast cell line (WI38). Additionally, exosomes isolated from PC3 cells have no effect on PC3 cells. This is also true for exosomes isolated from $\mathrm{H}_{2} \mathrm{O}_{2}$ induced senescent human lung cancer cells (A549). Analysis of exosome content by western blotting reveals the presence of PTEN in the exosome of lung cancer cells. Functional analysis of PTEN pathways in PC3 cells indicates the inactivation of Akt in exosome treated cells. Therefore, from our study we have concluded that exosomes secreted from A549 cells which contain functional PTEN, may be used for delivery of PTEN to cancer cells without functional PTEN.

\section{Introduction}

Exosomes are small sized extracellular vesicles surrounded by phospholipid bilayer and their size ranges are approximately $30-150 \mathrm{~nm}[1,2]$. Almost all kinds of cells are capable of secreting exosomes. While being released from the cells, exosomes carry some components such as proteins, mRNA, microRNA, siRNA, DNA etc. [3]. It has been found that exosomes are less immunogenic and non-cytotoxic to the recipient cells [4]. This makes them a potential carrier of drugs and other therapeutic agents to treat different types of diseases in recipient cells. Exosomes after fusion with recipient cells release the cargos into that cell. Thus, they play an important role in cell to cell communication [2]. The most important cargoes that exosomes carry are the proteins which can provide crucial information correlated with the physiological states of the cells from which exosomes originate. Proteins that are found in exosomes include membrane transport and fusion proteins such as annexins, flotillins, GTPases, heat shock proteins, tetraspanins, proteins involved in multivesicular body biogenesis, lactadherin, platelet derived growth factor receptor, transmembrane proteins and lysosome associated membrane protein-2B, as well as lipid-related proteins and phospholipases. These proteins can therefore be used as biomarkers for isolation and quantification of exosomes [1].

The phosphatase and tensin homolog, PTEN (also named MMAC1/TEP1), deleted on chromosome 10, is a major tumor suppressor protein. It negatively regulating phosphatidylinositol-3-kinase (PI3K), Akt, mTOR related signaling pathway [5]. It is well known that PTEN is often mutated in different types of human carcinoma. In prostate cancer cell (PC3) PTEN undergoes deletion mutation. While the lung carcinoma cells (i.e., A549 cell line) are PTEN positive cells [6]. Exosomes often act as promoters of tumorigenesis. Cancer cells secrete exosomes which may participate in modulating their signaling pathways associated with tumor promotion, immune escape, drug resistance, antiapoptotic features etc. 
Due to their role as communicator and carrier of different cargo, they mediate crosstalk between cells in tumor microenvironment stimulating tumor development [7]. Additionally, cancer cell derived exosomes can promote apoptosis induction and thereby inhibit tumor growth. For example, exosomes secreted by HEK293 and HT-1080 can suppress growth and proliferation of p53-deficient cells [8]. Senescent cells secrete exosomes which function as part of pro-tumorigenic Senescence-Associated Secretory Phenotype (SASP) [9]. However, it has also been found that exosomes derived from Mesenchymal stem cells promote the upregulation of P27kip1 as well as downregulation of Cdk2 pathway leading to cell cycle arrest [10]. Promotion of cancer [11,12] as well as induction of apoptosis [13] by SASP released by senescent fibroblasts has been reported. Thus, there are contrasting reports on the role of SASP in cellular proliferation and tumor promotion. Thus, potential applications of exosomes as a therapeutic agent may still be needed to explore further. The contains of therapeutic important materials in the exosome or secretome may be beneficial to the recipient cells. In view of this, we have isolated the exosome from cancer cells which have normal PTEN and delivered this exosome to PTEN deficient PC3 cells and found the growth arrest. One step further, we found growth arrest of PC3 cells when exposed to exosomes isolated from senescent cells. Thus, our observation has an important therapeutic implication in future.

\section{Materials And Methods}

\subsection{Antibodies and reagents}

Total Exosome Isolation Reagent (Invitrogen, 4478359), RPMI 1640 (Himedia, AT120), Dulbecco's Modified Eagle Medium (DMEM) (Himedia, AT007), Fetal bovine serum (FBS) (10270), Exosome depleted Fetal Bovine Serum (Gibco), Hydrogen peroxide 30\% (Merck,107209), Phosphate Buffer Saline (Himedia, TS1006), Dynabeads Protein A (Invitrogen, LT-02241), [3-(4,5-dimethylthiazoyl-2-yl)-2,5-

diphenyltetrazolium bromide] (MTT) purchased from SRL (India), Extraction buffer (Isopropanol Merck DA9P690159, TritonX-100 807428, Hydrochloric acid), Paraformaldehyde (Himedia, RM3660), Tween-20 (Amresco, CAS 9005-64-5), DAPI (Vectashield, H-1200-10), Senescence Beta-Galactosidase Staining Kit (Cell Signalling Technology, CAS 9860S), Low Melting Point Agarose (SRL, CAS 9012-36-6), Electrophoresis buffer ( $1 \mathrm{M}$ sodium EDTA, $300 \mathrm{mM} \mathrm{NaOH}$ ), Neutralizing buffer (0.4 M Tris, pH-7.5), EtBr $(20 \mu \mathrm{g} / \mathrm{mL})$, Lysis buffer (1\% Triton X-100, $50 \mathrm{mM} \mathrm{NaCl}, 50 \mathrm{mM} \mathrm{NaF}, 20 \mathrm{mM}$ Tris (pH 7.4), 1 mM EDTA, 1 $\mathrm{mM}$ EGTA, $1 \mathrm{mM}$ sodium vanadate, $0.2 \mathrm{mM}$ Phenylmethanesulfonylfluoride fluoride (PMSF), 0.5\% NP-40, protease and phosphatase inhibitor), Glycine (CAS 56-40-6), Tris base (CAS 77-86-1), SDS (CAS 151-21-3), Acrylamide/bis-acrylamide (79-06-1), polyvinylidene difluoride membrane, $5 \%$ non-fat dry milk, $0.05 \%$ Tween-20, 20mM Tris-Cl, pH 7.6 (TBS-T), Protease inhibitor cocktail (M250-1 ml), Phosphatase inhibitor cocktail II (GX-0211AR) were from Genetix, India, Bovine serum albumin (BSA) (Sigma Aldrich A9418), Bradford's reagent (Sigma Aldrich B6916), Flotilin 1 rabbit mAb (Cell Signalling Technology, D2V7J), Annexin V (Cell Signalling Technology, 8555T), CD9 Rabbit mAb (Cell Signalling Technology, D801A), CD63 rabbit polyclonal antibody (Santa Cruz Biotechnology, sc-15363), PTEN rabbit monoclonal antibody (Abcam, ab32199), GAPDH rabbit polyclonal antibody (Bio-Bharati LifeScience, BB-AB0060), Akt rabbit polyclonal antibody (Cell Signalling Technologies, 9272s), Phospho-Akt rabbit monoclonal antibody (Cell 
Signalling Technologies, 4058s), H3K9me3 rabbit polyclonal antibody (Abcam ab8898), $\mathrm{Y}$-H2AX Rabbit polyclonal antibody (Santa Cruz Biotechnology, sc-101696). Anti-mouse or anti-rabbit IgG conjugated with horseradish peroxidase (HRP, 1:1000, Bangalore Genei, India) and anti-rabbit IgG conjugated with FITC and anti-mouse IgG conjugated with Texas Red (1:200, Santa Cruz Biotechnology) were used as secondary antibody.

\subsection{Cell culture and treatment}

PC-3 (Human prostate carcinoma cells), A549 (Human lung carcinoma cells), HeLa (Cervical carcinoma cells) and WI-38 (Normal lung fibroblast cells) were purchased from the National Center for Cell Science, (Pune, India) and maintained at $37^{\circ} \mathrm{C}, 5 \% \mathrm{CO} 2$ and $95 \%$ relative humidity (RH) in RPMI 1640 and DMEM media respectively, supplemented with 10\% FBS and/or 10\% Exosome depleted FBS, penicillinstreptomycin (100 units $/ \mathrm{ml}$ ) and, amphotericin-B (anti-fungal). Cells were seeded overnight in two $60 \mathrm{~mm}$ culture plates in media supplemented with $10 \%$ FBS. Then, media of both the plates were withdrawn and fresh media supplemented with $10 \%$ exosome depleted FBS were added to the plates. One of the plates was treated with hydrogen peroxide $(\mathrm{H} 2 \mathrm{O} 2)(150 \mu \mathrm{M}$ for $48 \mathrm{~h})$ and the other was kept as untreated.

\subsection{Isolation of Exosome}

Exosome isolation was done using kit reagent. The cell culture medium was collected according to the manufacturer's protocol. After the isolation, the exosome pellets were finally dissolved in 1X PBS and stored at $-80^{\circ} \mathrm{C}$ for further uses.

\subsection{Dynamic Light Scattering (DLS)}

The particle diameter and size distribution of the isolated samples were measured by DLS instrument (ZetaSizer-HT Malvern). The Z-average values and the particle dispersion index (PDI) values were obtained for comparative study of the sizes of the isolated samples.

\subsection{Scanning Electron Microscopy (SEM)}

Pellets containing exosomes from healthy cells and senescent cells were fixed with $2 \%$ paraformaldehyde for 10 mins. The samples were then added to cleaned silicon chips, followed by sonication in ethanol for 5 mins and immobilized after drying under a laminar airflow. To make the surface conductive, a coating of 2-5 nm gold-palladium alloy was applied by sputtering (Argon as gas for plasma) before imaging by scanning electron microscopy (Inspect FEl, Netherlands). Analysis of exosome sizes was done using the SEM images via ImageJ software.

\subsection{MTT assay}

The inhibition of cell growth was measured by MTT assay. In brief, the human cell lines PC3, WI38 respectively seeded in 12 well plates. PC3 cells was treated with exosomes $(100 \mu \mathrm{g} / \mathrm{mL})$ isolated from mock treated A549 and Hela cells respectively and treated with exosomes $(100 \mu \mathrm{g} / \mathrm{mL})$ isolated from A549 cells treated with $\mathrm{H}_{2} \mathrm{O}_{2}$. Similarly WI38 cell was treated with exosomes $(100 \mu \mathrm{g} / \mathrm{mL})$ isolated from mock treated and $\mathrm{H}_{2} \mathrm{O}_{2}$ treated A549cells. In another set PC3 cells was treated with exosomes 
$(100 \mu \mathrm{g} / \mathrm{mL})$ isolated from mock treated and $\mathrm{H}_{2} \mathrm{O}_{2}$ treated PC3 cells. MTT reagents are incubated for $3 \mathrm{~h}$ at $37^{\circ} \mathrm{C}$. The resulting formazan crystals were then dissolved in MTT solubilization buffer and the absorbance were taken with a UV-vis spectrophotometer (Hitachi) at 570.

\subsection{Senescence associated (SA)- $\beta$-galactosidase staining assay}

A549 cells were seeded overnight on cover slip. Next day, cells were washed with 1X PBS and were replaced with fresh medium. One set of cells were kept as control and the other set was treated with $\mathrm{H} 2 \mathrm{O} 2(150 \mu \mathrm{M})$ and incubated for $2 \mathrm{~h}$. After the completion of the incubation period the cells were stained with a senescence associated beta-galactosidase staining kit (Cell Signaling Technology, CAS 9860S). Finally, the stained coverslips were mounted on grease free slides using $50 \%$ glycerol. Then, the slides were examined under a bright field of fluorescent microscope for detecting the senescent cells under $10 \mathrm{X}$ magnification.

\subsection{Fluorescence microscopy}

A549 cells were seeded overnight on cover slip. The next day, cells were washed with 1X PBS and media were replaced by fresh medium. Then, one set of cells were kept as control and the other set was treated with $\mathrm{H} 2 \mathrm{O} 2(150 \mu \mathrm{g} / \mathrm{mL})$ and incubated for $48 \mathrm{~h}$. The cells, after being incubated were fixed with $4 \%$ paraformaldehyde solution for $15 \mathrm{~min}$ and permeabilized with $0.2 \%$ Triton X-100 at $4^{\circ} \mathrm{C}$, for another 15 min. The cells were again washed followed by addition of $5 \%$ blocking solution ( $0.5 \%$ FBS in $1 \times$ PBS) for $1 \mathrm{~h}$ at room temperature followed by overnight incubation with $\mathrm{H} 3 \mathrm{k} 9 \mathrm{me} 3$ and $\mathrm{\gamma H} 2 \mathrm{AX}$ antibody in wash buffer $(0.5 \%$ FBS and $0.05 \%$ Tween 20 in $1 \times$ PBS $)$ at $4^{\circ} \mathrm{C}$. Next day, the cells were washed with wash buffer and followed by addition of anti-rabbit IgG conjugated with FITC and anti-mouse IgG conjugated with Texas Red antibodies for $1 \mathrm{~h}$ at room temperature (dark condition). After washing, cells containing coverslips were mounted with the mounting solution containing DAPI (4,6-diamidino-2-phenylindole, Vector Laboratories, USA) and examined under a fluorescence microscope (100X objectives, Leica, Germany).

\subsection{Western blotting}

Whole cell lysates were prepared by using cell lysis buffer $(50 \mathrm{~mm}$ Tris- $\mathrm{HCl} \mathrm{pH} \mathrm{8.0,100} \mathrm{mM} \mathrm{NaCl}, 0.5 \%$ NP-40, $1 \mathrm{mM}$ dithiothreitol, $2 \mu \mathrm{g} / \mathrm{ml}$ aprotinin, $2 \mu \mathrm{g} / \mathrm{ml}$ leupeptin, protease and phosphatase inhibitor). The protein concentrations of the isolated exosomes and whole cell lysates were also evaluated by Bradford assay. The cellular extracts and exosomes were solubilized in a protein loading buffer, boiled for 5 min, and electrophoresed on a 10\% SDS-polyacrylamide gel. Proteins were then transferred to methanol activated polyvinylidene difluoride (PVDF) membrane. The membrane then is blocked in $5 \%$ non-fat dry milk in $0.05 \%$ Tween-20 in $20 \mathrm{mM}$ Tris- $\mathrm{Cl}$, pH 7.6 (TBS-T) for $1 \mathrm{~h}$ at room temperature. After overnight incubation with appropriate primary antibody, the membrane was washed with TBST followed by reincubation with secondary antibodies conjugated with horseradish peroxidase (HRP) for $1 \mathrm{~h}$ at room 
temperature. Proteins were detected by Advansta ECL western blotting detection reagent. The intensity of each band was measured by ImageJ software.

\subsection{Comet Assay}

PC3 cells were seeded in $35 \mathrm{~mm}$ cell culture plates overnight. Next day, keeping one set without being treated and other two sets were treated with exosomes derived from mock treated and H2O2 treated A549 cells, respectively for $48 \mathrm{~h}$. Then, the cells were trypsinized and centrifuged. The pellets were washed in 1X PBS. PC3 cell pellet was mixed with $37^{\circ} \mathrm{C} 0.8 \%$ low-melting point agarose and transferred to normalmelting point agarose coated slides. The slides were mounted with cover slip and incubated at $4^{\circ} \mathrm{C}$ for solidification. Then, the slides were immersed in lysis buffer $(2.5 \mathrm{mM} \mathrm{NaCl}, 100 \mathrm{mM}$ EDTA, $10 \mathrm{mM}$ Tris, $10 \%$ DMSO and $1 \%$ Triton $\mathrm{X}-100)$ for $1 \mathrm{~h}$ at $4^{\circ} \mathrm{C}$. After washing with neutralizing buffer $(0.4 \mathrm{M} \mathrm{Tris,} \mathrm{pH}-$ 7.5), the slides were immersed into electrophoresis buffer within an electrophoresis tank. Electrophoresis was carried out at $25 \mathrm{~V}$ and $300 \mathrm{~mA}$ for $20 \mathrm{~min}$. After which, the slides were washed with a neutralizing buffer for $40 \mathrm{~min}$. After washing the slides were stained with EtBr solution and kept in the dark for 20 min. The stained slides were washed with 1X PBS and were finally mounted with cover slips by using $50 \%$ glycerol as mounting media and scored using a fluorescence microscope (Leica, Germany). \% of tail DNA (DNA fragment percent in the tail) were scored with the help of Komet Assay Software 5.5 at 40X magnification.

\subsection{Cell damage detection}

\section{a) Analysis of nuclear morphology by DAPI staining}

Cells were seeded on cover slips. Next day, keeping one set as control, the other set cells were treated and then incubated for $48 \mathrm{~h}$. Then, the cells were washed with 1X PBS for 2-3 times and the cell containing coverslips were mounted on grease free slides with DAPI stain and finally observed under $100 \mathrm{X}$ objective of fluorescent microscope.

b) Cell staining with Hoechst dye

Cells were seeded on cover slips. Next day, cells were treated and incubated for $48 \mathrm{~h}$ keeping one set as control. The cells were washed with 1X PBS for 2 to 3 times. Then the cells were stained with 1X Hoechst dye solution, followed by 15 mins of incubation in dark condition, at room temperature. After washing with 1 X PBS for 1 to 2 times, cells were mounted with $50 \%$ glycerol on grease free slides and were examined under fluorescent microscope at $100 \mathrm{X}$ magnification. 


\subsection{Cell cycle analysis}

PC3 cells were seeded in $60 \mathrm{~mm}$ cell culture plates overnight. At $24 \mathrm{~h}$ after seeding, keeping one set without being treated and other two sets were treated with $100 \mu \mathrm{g} / \mathrm{mL}$ of exosomes derived from mock treated and $\mathrm{H} 2 \mathrm{O} 2$ treated $\mathrm{A} 549$ cells, respectively for $48 \mathrm{~h}$. Then, the cells were trypsinized and centrifuged resulting in the formation of pellets. The pellets were washed with 1X PBS and fixed with chilled $70 \%$ ethanol and kept for overnight at $-20^{\circ} \mathrm{C}$. Prior to stain with $50 \mu \mathrm{g} / \mathrm{mL}$ propidium iodide (PI, Invitrogen), the cells were incubated for 30 mins with $100 \mu \mathrm{g} / \mathrm{mL}$ of RNAse A (SRL, India) at $37^{\circ} \mathrm{C}$. The cell cycle was analysed with flow cytometry.

\subsection{Statistical analysis}

A student's t-test was used to calculate the statistical significance of changes between the groups. $\mathrm{P} \otimes$ 0.05 and 0.005 was considered as statistically significant. Data analysis was performed using the Origin Pro v.8 software (Origin Lab).

\section{Results}

\subsection{Characterization of exosome by DLS and SEM}

The size of exosomes derived from mock-treated and $\mathrm{H}_{2} \mathrm{O}_{2}$ treated $\mathrm{A} 549$ cells were investigated using FESEM, as shown in Fig. 1a. The particle size of the exosome isolated from mock-treated cells was observed to be $60 \pm 10 \mathrm{~nm}$ while the exosome isolated from cells treated with $\mathrm{H}_{2} \mathrm{O}_{2}$ showed slight increase in size which is $85 \pm 15 \mathrm{~nm}$ with no change in surface morphology in both the cases. Additionally, the size distribution study by DLS also revealed a slightly larger size of exosome isolated from $\mathrm{H}_{2} \mathrm{O}_{2}$ treated cells as shown in Fig. $1 \mathrm{~b}$ and $1 \mathrm{c}$. Additionally, the purity of the exosome was analysed by western blotting as shown in Fig. 1d. Presence of CD9, Flotillin 1, Annexin V and CD63 indicated the purity of isolated exosomes.

\subsection{Cell survivability}

Effect of exosomes on cell growth was explored on human prostate carcinoma cell lines PC3 and SV40 transformed normal lung fibroblast cell line WI-38. Survivability percentage of PC3 cells was calculated after treating them with exosomes derived from mock treated A549 cell line or Hela cells at different time intervals (Fig. $2 a$ and $2 b$ ). For both the cases survivability of PC3 cells significantly reduced ( $\left.{ }^{*} \otimes 0.05\right)$ to almost $80 \%$ after $24 \mathrm{~h}$ and $48 \mathrm{~h}$ intervals. But exosomes derived from mock treated and $\mathrm{H}_{2} \mathrm{O}_{2}$ treated PC3 cells when treated with PC3 cells itself, no change in survivability was observed in both the cases (Fig. 2c). Also, when normal fibroblast cell line Wl-38 was treated with exosome isolated from mock treated or $\mathrm{H}_{2} \mathrm{O}_{2}$ treated $\mathrm{A} 549$ cells, survivability of WI-38 cells remained the same (Fig. $2 \mathrm{~d}$ ). Thus the exosome secreted by A549 cells contains some factors which selectively inhibits growth of PC3 cells. 
Finally, when PC3 cells were treated with exosomes isolated from $\mathrm{H}_{2} \mathrm{O}_{2}$ treated (150 $\mu \mathrm{M}$, for $\left.2 \mathrm{~h}\right) \mathrm{A} 549$ cell line at different time intervals, the survivability significantly reduced to $60 \%\left({ }^{*} \otimes 0.05, * \star P \nabla 0.005\right)$ upto $48 \mathrm{~h}$ and after that the cell began to restore its growth indicating exhaustion of growth inhibitory effect of exosomes (Fig. 2e).

\subsection{Detection of PTEN in PC3 cells treated with A549 cell derived exosomes by western blotting}

Since aggressive nature of PC3 cells are due to the non-functional PTEN, we expect that exosome mediated delivery of PTEN may be responsible for their growth arrest. To prove this, we immunoblotted exosome content from both mock treated and $\mathrm{H}_{2} \mathrm{O}_{2}$ treated A549 cells with PTEN antibody. We found the presence of PTEN in both the cases (Fig. 3). In order to check the active function of PTEN transported by exosome in PC3 cell signaling pathways, we explored the signaling pathway associated with PTEN activity. Activation of PTEN resulted in dephosphorylation of Akt rendering the growth arrest as seen in Fig. 3. In PC3 cells after exposure to exosomes derived from mock treated or $\mathrm{H}_{2} \mathrm{O}_{2}$ treated A549 cells, PhosphoAkt is reduced compared to untreated cells. Furthermore, PTEN was detected when the PC3 cell was treated with exosome but no PTEN was detected in PC3 cell extract without any exosome treatment. Thus, this experiment confirmed exosome mediated delivery of catalytically active PTEN to PC3 cells.

\subsection{Cell cycle study}

Cell cycle arrest may be mediated through DNA damage which may lead to apoptosis. Thus, we first analysed the cell cycle of PC3 cells after exosome treatment. As seen in Fig. 4a the \% of sub G1 cells did not increase, indicating the absence of apoptotic cells, while the \% of S phase cells significantly decreased (*P $\otimes 0.05$ ) from 31.97 to 10 and 6.16 respectively in case of PC3 cells. Figure $4 \mathrm{~b}$ and $4 \mathrm{c}$ represent the bar graph of cell cycle and quantification of number of cells in each phase, respectively.

\subsection{Comet Assay}

Growth arrest of PC3 cells may be mediated by DNA damage induced by the factors present in the exosome. To address this, we performed comet assay and observed the DNA damage repair activity of PTEN in the PC3 cells treated with exosomes isolated from mock-treated and $\mathrm{H}_{2} \mathrm{O}_{2}$ treated A549 cells. DNA damage was observed in those cells without any exosome treatment and exosome treated PC3 cells showed no damage (Fig. 5a). The amount of damage was determined by \% of tail DNA. For control PC3 cells, cells treated with exosomes derived from $\mathrm{H}_{2} \mathrm{O}_{2}$ treated A549 cells and PC3 cells treated with exosomes derived from mock treated A549 cells, the \% of tail DNA was significantly found to be $18 \%$, $8.67 \%$ and $11 \%$ respectively as shown in Fig. 5b (**P $\otimes 0.005)$.

\subsection{Analysis of cellular senescence}

Thus functional PTEN delivered by exosome mediates temporary growth arrest in PC3 cells. Now both mock treated and $\mathrm{H}_{2} \mathrm{O}_{2}$ treated cells derived exosomes can induce growth arrest in PC3 cells. We only found a little larger exosome in $\mathrm{H}_{2} \mathrm{O}_{2}$ treatment. Thus, we wanted to determine the fate of $\mathrm{H}_{2} \mathrm{O}_{2}$ treated 
A549 cells. In simple DAPI and Hoechst staining of A549 cells no change in nuclear morphology was observed (Fig. 6a and 6b). Since no apoptosis was observed in $\mathrm{A} 549$ cells after $\mathrm{H}_{2} \mathrm{O}_{2}$ treatment (data not shown) the possibility of senescence in A549 cells after treatment with $\mathrm{H}_{2} \mathrm{O}_{2}$ was explored. Senescence associated beta galactosidase assay (Fig. 6c) showed induction of senescence in A549 cells after treatment with $\mathrm{H}_{2} \mathrm{O}_{2}(150 \mu \mathrm{M})$ for $2 \mathrm{~h}$. Quantitatively, the \% of senescent cells significantly increases ( ${ }^{*} \mathrm{P}$ 0.05) (Fig. 6 d) in $\mathrm{H}_{2} \mathrm{O}_{2}$ treated $A 549$ cells at $2 \mathrm{~h}$ (56.75 \pm 3.14 ) compared to mock treated $A 549$ cells (7.6 \pm 1.32). Additionally, senescence associated heterochromatin foci were also tested for $\mathrm{H}_{2} \mathrm{O}_{2}$ treated cells by observing foci of $\mathrm{H} 3 \mathrm{~K} 9 \mathrm{me} 3$ (Fig. $6 \mathrm{e}$ ) and $\mathrm{Y}-\mathrm{H} 2 \mathrm{AX}$ foci (Fig. $6 \mathrm{~g}$ ). Here we also observed (Fig. $6 \mathrm{f}$ and

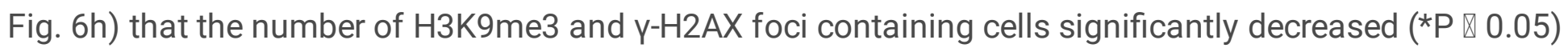
in $\mathrm{H}_{2} \mathrm{O}_{2}$ treated cells $(21.02 \pm 2.4$ and $49.55 \pm 3.86)$ compared to mock treated cells $(54.61 \pm 2.2$ and $80.71 \pm 3.5)$.

\section{Discussion}

Exosomal cargo contains numerous factors including DNA, proteins, RNA etc [3]. Here we have seen that exosomes from lung fibroblast cells can induce growth arrest in PC3 cells. PC3 cells are implicated as deleted mutations in PTEN and lack of PTEN are supposed to be responsible for its abnormal growth [14]. Thus, delivery of functional PTEN may be a potential therapeutic approach to arrest growth of cells having downregulated PTEN. Exosome mediated delivery of different agents are emerging as a potential approach as it is non immunogenic $[4,15]$. Here we have seen the exosomes derived from A549 cells and HeLa cells are capable of inhibiting growth of PC3 cells. While most of the studies indicated the tumour promoting activity of exosomes secreted by various cells $[1,7]$, but some reports available where exosomes seem to inhibit tumour growth [16]. The protein composition of exosomes is not unique and remains to be explored further. But the content of the exosome varies between cells from which they originate proteins which are no longer necessary for their survival or excess protein production due to mutation [17]. Thus, exosomal content was suggested to be biomarkers of different diseases. Though, due to their endosomal origin they contain some marker proteins (flotillin, CD9, CD63 etc.) which are found in exosomes from different cells [1]. Here also we have seen the presence of some of these proteins in our exosome. Here, at first, we found that exosomes from lung cancer cells can inhibit the growth of prostate cancer cells specifically and have no effect on normal lung fibroblast. Also exosome from prostate cancer cells (PC3) has no effect on PC3 cells. We initially thought that under stress conditions lung cancer cells may act differentially and thus can change the exosomal content and impart different effects on PC3. But even under stress conditions, exosomes secreted by A549 cells can arrest the growth of PC3. Since some miRNA carried by exosomes may alter the DNA damage response pathway [18], we explored the possibility also and found that was not the case. Induction of apoptosis by exosomal content is also reported [14] but in our case, the exosome cannot induce apoptosis in PC3 cells. Genetic data reveals that PC3 cells have deleted PTEN where lung cancer cell A549 has mutated Ras protein $[14,19]$. This prompted us to look for PTEN in the A549 derived exosomes. Surprisingly even under stress conditions, lung cancer cells can secrete exosomes containing PTEN. Functional analysis of PTEN was also carried out. The most potential approach in this case is to analyse the PI3K pathway, 
where we found delivered PTEN in PC3 cells can inhibit the phosphorylation of Akt. Usually in cancer cells Akt is hyperactivated by phosphorylation and induces tremendous cell growth even under DNA damage conditions rendering genomic instability [20]. In our case we also found dephosphorylation of Akt in PC3 cells indicating functionally active PTEN was delivered and Akt mediated signaling for the growth of PC3 cells is perturbed. It has been reported earlier that cancer cells use a noble mechanism in the downregulation of PTEN [21-23]. It seems likely that A549 cells while having wild type PTEN may use exosomal pathways to overcome the tumor suppressor activity of PTEN. Thus functional PTEN, even it is present in lung cancer cells, may not impose growth arrest activity on A549 cells. On the other hand, senescence induced by $\mathrm{H}_{2} \mathrm{O}_{2}$ in A549 cells resulted in excretion of PTEN and must be accompanied by some other factor associated with small extracellular vehicle (sEV) responsible for changing several activities of recipient cells. In our case we have seen larger volume of sEV compared to untreated A549 cells indicating the amount of content increases under senescence condition (Fig. 1a and 1c). But the growth arrest is temporary as after $72 \mathrm{~h}$ of treatment the inhibitory effect vanishes perhaps due to exhaustion of imported PTEN. Additionally, PTEN is emerging as a DNA repair protein and responsible for maintaining genomic stability. In our study we have seen that exosome treated PC3 cells have less amount of tail DNA in comet assay (Fig. 5b). Thus, A549 cells derived from the exosome contain functionally active PTEN which is likely to be responsible for growth inhibition of PC3 cells. These findings have important therapeutic implications to treat prostate cancer cells or any cancer associated with dysfunction PTEN. To observe the change in nuclear morphology after treating the exosome donor A549 cell with $\mathrm{H}_{2} \mathrm{O}_{2}$ we used staining procedures with DAPI and Hoechst dye. In DAPI staining, the characteristic change in nuclear morphology was observed in cells treated with $\mathrm{H}_{2} \mathrm{O}_{2}$ while the nucleus of the mock treated cells appeared intact (Fig. 6a). The heterochromatin foci which is observed in case of senescent cells due to staining with Hoechst dye was detected in $\mathrm{H}_{2} \mathrm{O}_{2}$ treated $\mathrm{A} 549$ cells and were absent in mock treated $\mathrm{A} 549$ cells (Fig. 6b). Exosomes produced from $\mathrm{H}_{2} \mathrm{O}_{2}$ treated lung cancer (A549) senescent cells transfer PTEN in PTEN null (PC3) cells resulting in cell growth arrest as long as $48 \mathrm{~h}$. PTEN, if inactivated selectively and temporarily induces hyperactivation of signalling pathway, leading to cellular senescence as reported by Alimonty et. Al [24]. Exosome is also a constituent of senescence associated secretory phenotype (SASP) along with a multitude of proteins such as growth factor, inflammatory cytokines [24]. Cell survivability in PTEN null cells (PC3) increases after $48 \mathrm{~h}$ which may be concluded by the fact that PTEN derived from exosomes of senescent cells may be selectively inactive which, on meeting the growth factor present within exosomes, induces cell survivability.

\section{Conclusion}

From our study it can be concluded that exosomes released from senescent cells can express PTEN and it also delivers PTEN into PTEN-null cancer cells. Expression of PTEN in the cancer cell can suppress the proliferation by $\mathrm{G} 1$ cell cycle arrest. Therefore, this study can open an anticancer strategy by improving the functional dose of PTEN.

\section{Declarations}


Data availability

The authors confirm that the data supporting the findings of this study are available within the article.

\section{Acknowledgements}

This work is financially supported by the Rashtriya Uchchatar Shiksha Abhiyan (RUSA) 2.0 (Reference No. R-11/400/2019, Date:19/4/19) and Department of Science and Technology and Biotechnology (Sanction no.BT/ST/P/S \& T/2G-13/2017), Govt. of West Bengal.

\section{Author information}

\section{Affiliations}

Department of Life Science and Biotechnology, Jadavpur University, Kolkata 700032, West Bengal, India Sougata Ghosh Chowdhury, Rachayeeta Ray \& Parimal Karmakar

\section{Department of Microbiology, Maulana Azad College, Kolkata 700013, West Bengal, India}

Debalina Bhattacharya

\section{Contributions}

PK conceived the project, designed the experiments. SGC and RR performed all of the experiments, wrote the manuscript and both equally contributed. DB analyzed the data. All authors have read and approved the manuscript.

\section{Corresponding author}

Correspondence to Parimal Karmakar.

Ethics declarations

\section{Conflict of interest}

All authors declare that there are no conflicts of interest.

\section{Ethical approval}

This article does not contain any studies with human and animal subjects performed by any of the authors.

\section{Consent for publication}


All the authors approved the publication.

\section{References}

1. Li P, Kaslan M, Lee SH, Yao J, Gao Z. Progress in Exosome Isolation Techniques. Theranostics. 2017;7(3):789-804. https://doi.org/10.7150/thno.18133.

2. Wu M, Ouyang Y, Wang Z, Zhang R, Huang PH, Chen C, Li H, Li P, Quinn D, Dao M, Suresh S, Sadovsky Y, Huang TJ. Isolation of exosomes from whole blood by integrating acoustics and microfluidics. Proceedings of the National Academy of Science United Stetes of America. 2017; 114(40) 1058410589. https://doi.org/10.1073/pnas.1709210114.

3. Albero MS, Navascués N, Mendoza G, Sebastián V, Arruebo M, Duque PM, Santamaria J. Exosome origin determines cell targeting and the transfer of therapeutic nanoparticles towards target cells. Journal of Nanobiotechnology. 2019;17(1):16. https://doi.org/10.1186/s12951-018-0437-z.

4. O'Loughlin AJ, Woffindale CA, Wood MJA. Exosomes and the emerging field of exosome-based gene therapy. Curr Gene Ther. 2012;12(4):262-74. https://doi.org/10.2174/156652312802083594.

5. Chen CY, Chen J, He L, Stiles BL. PTEN: Tumor Suppressor and Metabolic Regulator. Frontiers in Endocrinology (Lausanne). 2018; 9 338. https://doi.org/ 10.3389/fendo.2018.00338.

6. Bi L, Yan X, Yang Y, Qian L, Tian Y, Mao JH, Chen W. The component formula of Salvia miltiorrhiza and Panax ginseng induces apoptosis and inhibits cell invasion and migration through targeting PTEN in lung cancer cells. Oncotarget. 2017;8:101599-613. https://doi.org/10.18632/oncotarget.21354.

7. Milman N, Ginini L, Gil Z. Exosomes and their role in tumorigenesis and anticancer drug resistance. Drug Resist Updates. 2019;45:1-12. https://doi.org/10.1016/j.drup.2019.07.003.

8. Burdakov VS, Kovalev RA, Pantina RA, Varfolomeeva EY, Makarov EM, Filatov MV. Exosomes Transfer p53 between Cells and Can Suppress Growth and Proliferation of p53-Negative Cells. Cell Tissue Biology. 2018;12(1):20-6. https://doi.org/10.1134/S1990519X18010030.

9. Jakhar R, Crasta K. Exosomes as Emerging Pro-Tumorigenic Mediators of the SenescenceAssociated Secretory Phenotype. Int J Mol Sci. 2019;20(10):2547. https://doi.org/10.3390/ijms20102547.

10. Lee S, Kim S, Chung H, Moon JH, Kang SJ, Park CG. Mesenchymal stem cell-derived exosomes suppress proliferation of T cells by inducing cell cycle arrest through p27kip1/Cdk2 signaling. Immunol Lett. 2020;225:16-22. https://doi.org/10.1016/j.imlet.2020.06.006.

11. Krtolica A, Parrinello S, Lockett S, Desprez PY, Campisi J. Senescent fibroblasts promote epithelial cell growth and tumorigenesis: a link between cancer and aging. Proceedings of the National Academy of Science United Stetes of America. 2001; 98(21) 12072-12077. https://doi.org/10.1073/pnas.211053698.

12. Liu D, Hornsby PJ. Senescent human fibroblasts increase the early growth of xenograft tumors via matrix metalloproteinase secretion. Can Res. 2007;67(7):3117-26. https://doi.org/10.1158/0008- 
5472.CAN-06-3452.

13. Vjetrovic J, Shankaranarayanan P, Parra MAM, Gronemeyer H. Senescence-secreted factors activate Myc and sensitize pretransformed cells to TRAIL-induced apoptosis. Aging Cell. 2014;13(3):487-96. https://doi.org/10.1111/acel.12197.

14. Malla B, Zaugg K, Vassella E, Aebersold DM, Pra AD. Exosomes and Exosomal MicroRNAs in Prostate Cancer Radiation Therapy. Int J Radiat Oncol Biol Phys. 2017;98(5):982-95. https://doi.org/10.1016/j.ijrobp.2017.03.031.

15. Ha D, Yang N, Nadithe V. Exosomes as therapeutic drug carriers and delivery vehicles across biological membranes: current perspectives and future challenges. Acta Pharmaceutica Sinica B. 2016;6(4):287-96. https://doi.org/10.1016/j.apsb.2016.02.001.

16. Mao L, Li X, Gong S, Yuan H, Jiang Y, Huang W, Sun X, Dang X. Serum exosomes contain ECRG4 mRNA that suppresses tumor growth via inhibition of genes involved in inflammation, cell proliferation, and angiogenesis. Cancer Gene Ther. 2018;25(9-10):248-59. https://doi.org/10.1038/s41417-018-0032-3.

17. Yue B, Yang H, Wang J, Ru W, Wu J, Huang Y, Lan X, Lei C, Chen H. Exosome biogenesis, secretion and function of exosomal miRNAs in skeletal muscle myogenesis. Cell Prolif. 2020;53(7):e12857. https://doi.org/10.1111/cpr.12857.

18. Mo LJ, Song M, Huang QH, Guan H, Liu XD, Xie DF, Huang B, Huang RX, Zhou PK. Exosomepackaged miR-1246 contributes to bystander DNA damage by targeting LIG4. Br J Cancer. 2018;119(4):492-502. https://doi.org/10.1038/s41416-018-0192-9.

19. Schelch K, Vogel L, Schneller A, Brankovic J, Mohr T, Mayer RL, Slany A, Gerner C, Grusch M. EGF Induces Migration Independent of EMT or Invasion in A549 Lung Adenocarcinoma Cells. Frontiers in Cell Developmental Biology. 2021; 9 634371. https://doi.org/10.3389/fcell.2021.634371.

20. Liu Q, Turner KM, Yung WKA, Chen K, Zhang W. Role of AKT signaling in DNA repair and clinical response to cancer therapy. Neuro Oncology. 2014;16(10):1313-23. https://doi.org/10.1093/neuonc/nou058.

21. Tekcham DS, Gupta S, Shrivastav BR, Tiwari PK. Epigenetic Downregulation of PTEN in Gallbladder Cancer. Journal of Gastrointestinal Cancer. 2017;48(1):110-6. https://doi.org/10.1007/s12029-0179919-8.

22. Sun D, Lei W, Hou X, Li H, Ni W. PUF60 accelerates the progression of breast cancer through downregulation of PTEN expression. Cancer Management Research. 2019;11:821-30. https://doi.org/10.2147/CMAR.S180242.

23. Man X, Piao C, Lin X, Kong C, Cui X, Jiang Y. USP13 functions as a tumor suppressor by blocking the NF-kB-mediated PTEN downregulation in human bladder cancer. Journal of Experimental Clinical Cancer Research. 2019;38(1):259. https://doi.org/10.1186/s13046-019-1262-4.

24. Toso A, Revandkar A, Mitri DD, Guccini I, Proietti M, Sarti M, Pinton S, Zhang J, Kalathur M, Civenni G, Jarrossay D, Montani E, Marini C, Garcia-Escudero R, Scanziani E, Grassi F, Pandolfi PP, Catapano CV, Alimont A. Enhancing chemotherapy efficacy in Pten-deficient prostate tumors by activating the 
senescence-associated antitumor immunity. Cell Reports. 2014;9(1):75-89.

https://doi.org/10.1016/j.celrep.2014.08.044.

\section{Figures}
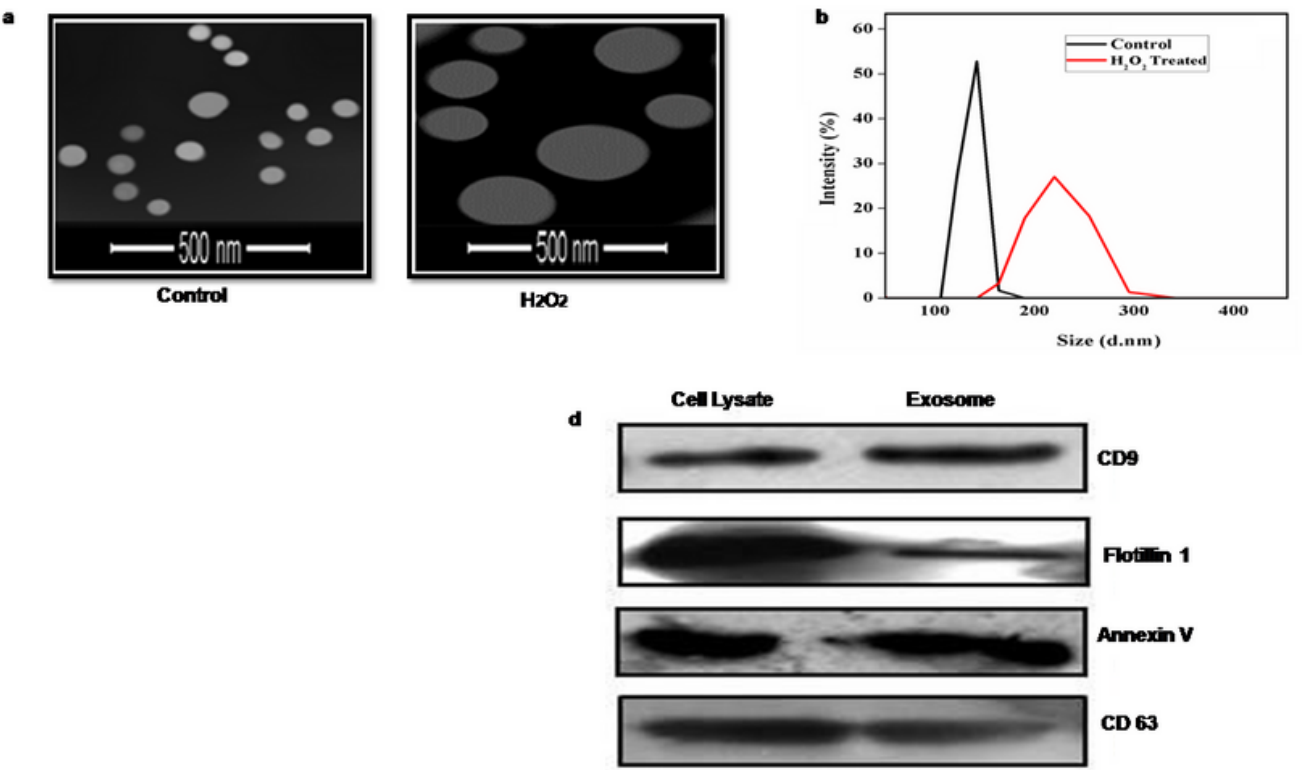

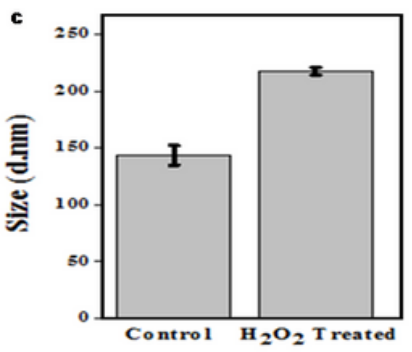

\section{Figure 1}

Physical characterization of A549 cell derived exosomes. FESEM image of exosomes derived from (a) Mock treated and $\mathrm{H} 2 \mathrm{O} 2$ treated A549 cells. Scales provided with the figures. Size determination of the isolated exosomes by Dynamic Light Scattering. (b) Linear graphical representation and (c) Columnar graphical representation of the A549 cell derived exosomes. Further DLS was performed to measure the average hydrodynamic particle sizes of the exosomes isolated from mock-treated and $\mathrm{H} 2 \mathrm{O} 2$ treated cells. The hydrodynamic radius (Rh) of the exosomes was found to be $150 \pm 10 \mathrm{~nm}$ and $200 \pm 15 \mathrm{~nm}$ respectively (Fig. 1C). Particle size obtained via DLS (average particle size) is much higher than that obtained via FESEM (actual particle size) due to DLS was performed in an aqueous medium, whereas FESEM was performed in a dry state. (d) Western blot analysis of cell lysate and A549 derived exosomes for detection of exosomal marker proteins, which showed the expression of CD9, Flotillin1, AnnexinV and CD63. 

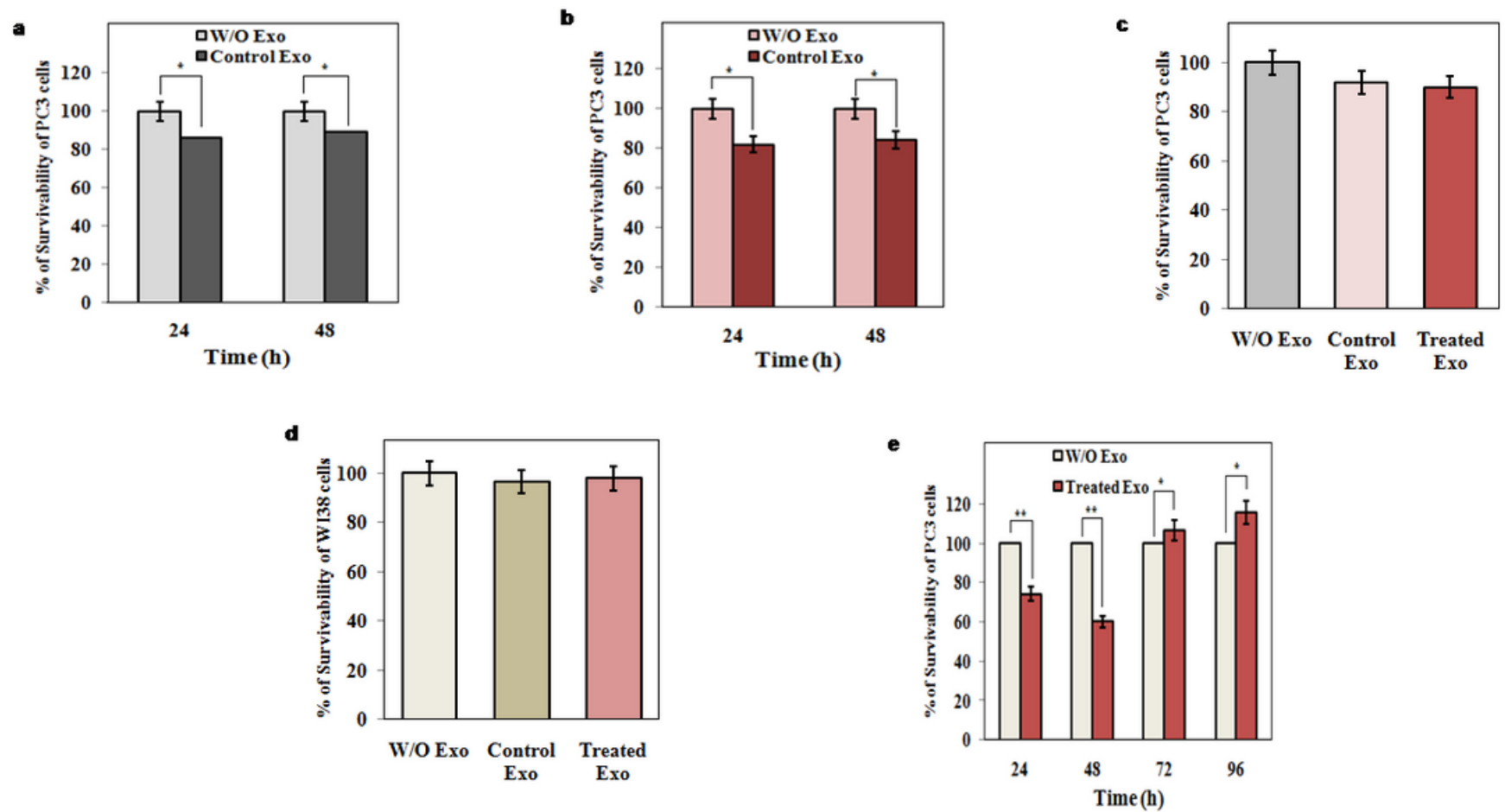

\section{Figure 2}

MTT results showing percentage of cell survival of (a) PC3 cell line treated with exosomes derived from mock treated A549 cell line (at different time intervals) (b) PC3 cell line treated with exosomes derived from mock treated HeLa cell line (at different time intervals). (c) PC3 cell line treated with exosomes derived from PC3 cell line which were both mock treated and treated with H2O2 (for 48 h) (d) Wl-38 cell line treated with exosomes derived from A549 cell line which were both mock treated and treated with $\mathrm{H} 2 \mathrm{O} 2$ (for 48h). (e) PC3 cell line treated with exosomes derived from $\mathrm{H} 2 \mathrm{O} 2$ treated A549 cell line (at different time intervals). Controls of each set were taken as $100 \%$. Values are the mean \pm SD of three independent experiments. ${ }^{*} \mathrm{P}<0.05,{ }^{*} \mathrm{P}<0.005$. 

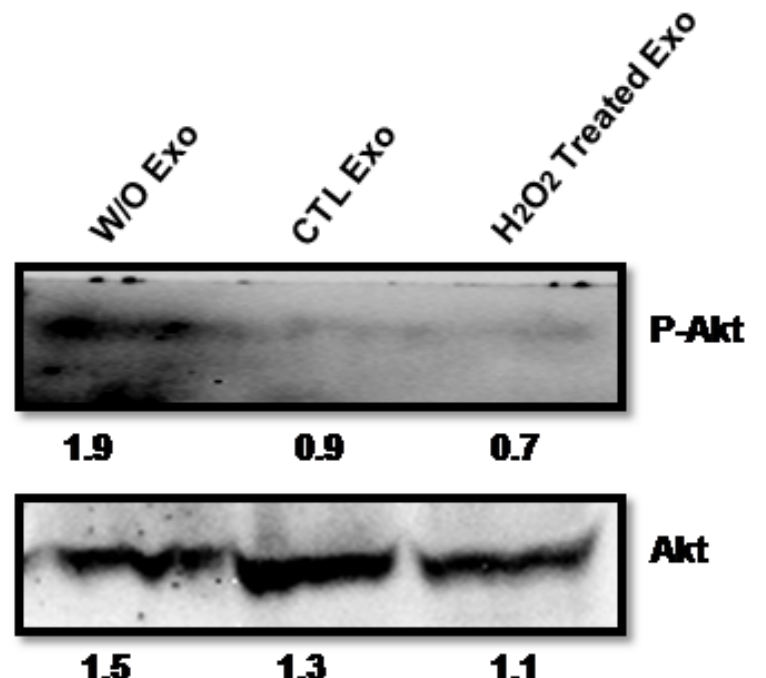

Akt

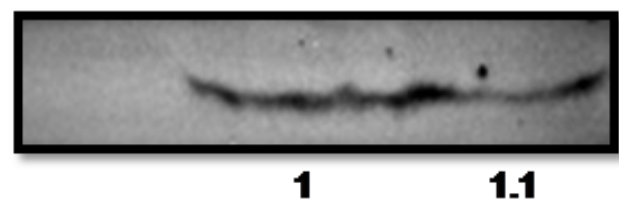

PTEN

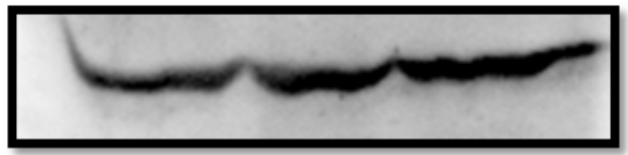

GAPDH

\section{Figure 3}

Western blot analysis of PC3 cells treated with exosomes isolated from wild type and $\mathrm{H} 2 \mathrm{O} 2$ treated A549 cells for presence of PTEN protein, using GAPDH as control and for detecting the activity of PTEN by determining the presence of p-Akt, using Akt as control. Band intensity was measured by ImageJ software. Control is normalized to 1 . 

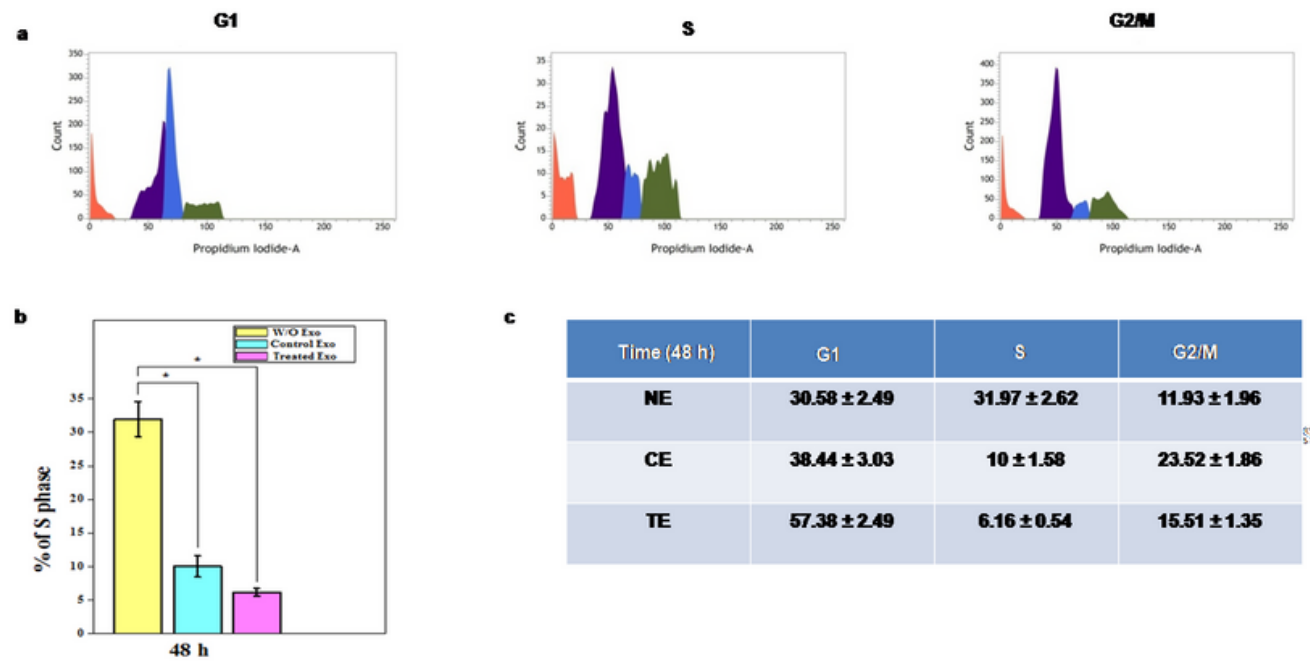

GYSM

\section{Figure 4}

Cell cycle analysis determined by Fluorescence-activated cell sorting (FACS). (a) showing the population of PC3 cells undergoing cell cycle arrest at phases $\mathrm{G} 1, \mathrm{~S}$ and $\mathrm{G} 2 / \mathrm{M}$ after treating them with A549 cell derived exosomes. (b) Graphical representation of the population of PC3 cells undergoing cell cycle arrest at S phase, after $48 \mathrm{~h}$ of $\mathrm{A} 549$ derived exosome treatment. Values are the mean \pm SD of three independent experiments. ${ }^{*} \mathrm{P}<0.05$. (c) Table showing percentage of PC3 cells undergoing cell cycle arrest at different phases after $48 \mathrm{~h}$ after treatment with A549 cell derived exosomes. NE: cells without any exosome treatment, CE: cells treated with exosomes derived from wild type A549 cells, TE: cells treated with exosomes derived from $\mathrm{H} 2 \mathrm{O} 2$ treated $\mathrm{A} 549$ cells. 
a

WIO Exo
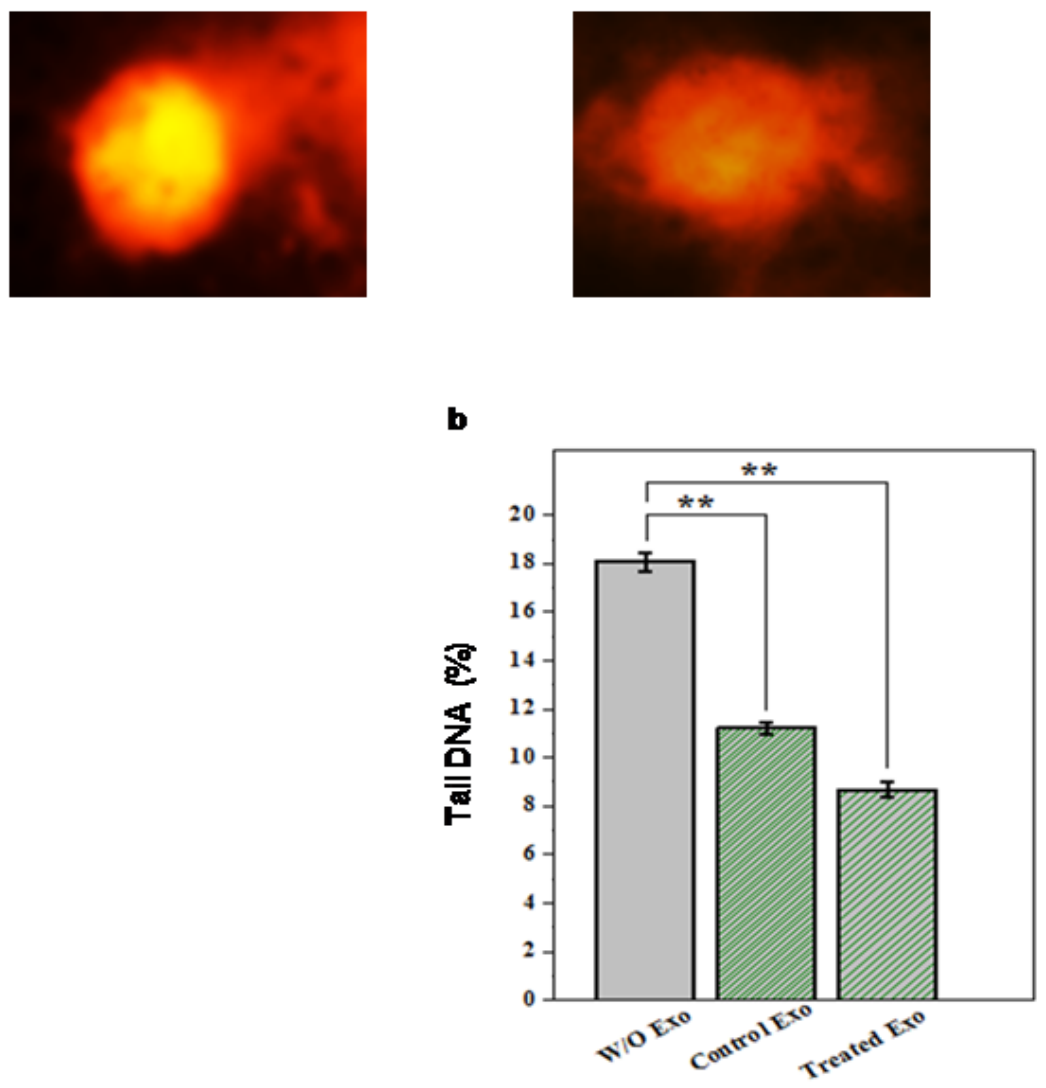

Treated Exo

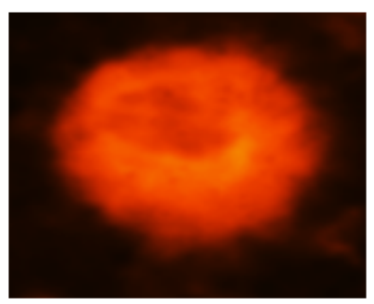

Figure 5

(a) Representative photomicrographs of comet assay of PC3 cells. DNA damage in nucleus of PC3 cells without exosome treatment, after $48 \mathrm{~h}$ treatment with exosome from mock treated A549 cells and with exosome from $\mathrm{H} 2 \mathrm{O} 2$ treated A549 cells. (b) Graph represents the percentage of tail DNA (measured by Komet 5.5 software). 40 nuclei per slide were counted. Values are the mean \pm SD of three independent experiments. ${ }^{* \star} \mathrm{P}<0.005$. 

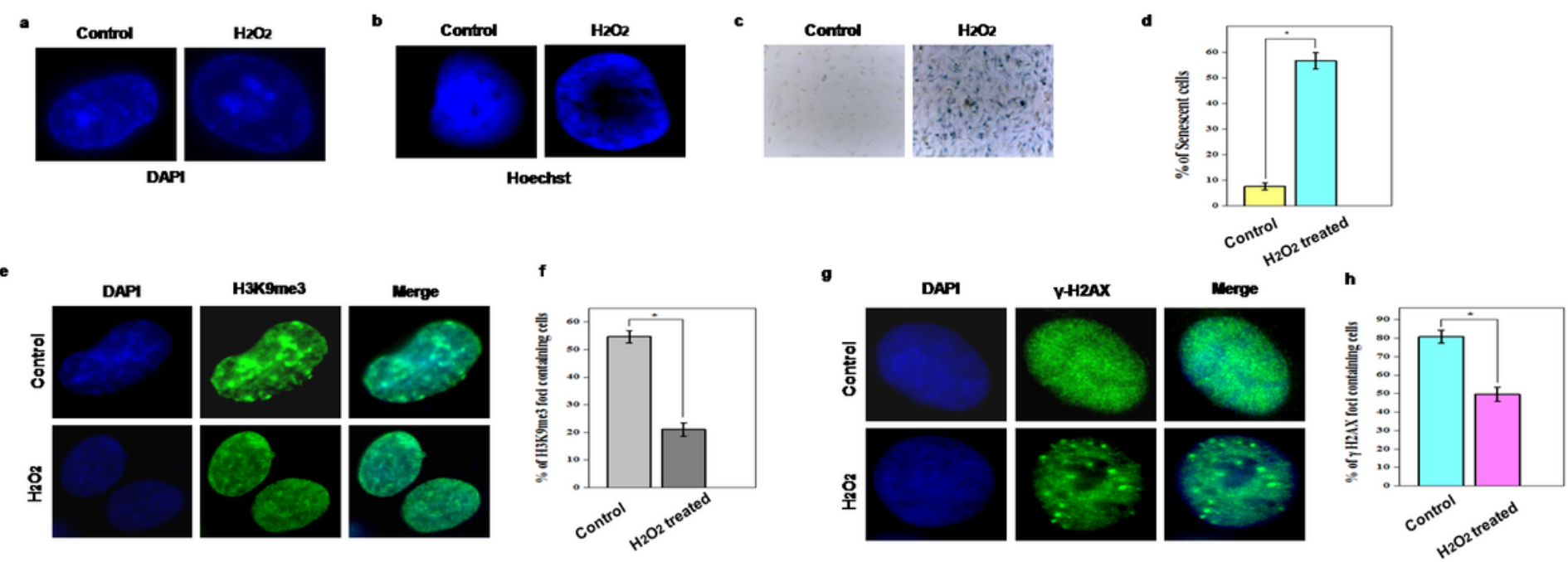

\section{Figure 6}

Nuclear morphology analysis through (a) DAPI staining and (b) Hoechst staining of exosome donating A549 cells. Detection of Senescence in exosome donating A549 cells. (c) A549 cells were stimulated with $\mathrm{H} 2 \mathrm{O} 2(150 \mu \mathrm{M})$ for $2 \mathrm{~h}$ and stained for detecting the activity of senescence-associated (SA)- $\beta$ galactosidase. (d) Quantitative results for the percentage of senescent cells stained with SA- $\beta$ galactosidase. Values are the mean \pm SD of three independent experiments. ${ }^{*} P<0.05$. Representative photomicrographs of $\mathrm{H} 3 \mathrm{~K} 9 \mathrm{me} 3, \mathrm{y}-\mathrm{H} 2 \mathrm{AX}$ foci of A549 cells. (e) Representative microscopic image of mock treated and $\mathrm{H} 2 \mathrm{O} 2$ treated $\mathrm{A} 549$ cells detected by H3K9me3. (f) Representative quantitative data of $\mathrm{H} 3 \mathrm{~K} 9 \mathrm{me} 3$ foci containing cells. Values are the mean $\pm \mathrm{SD}$ of three independent experiments. ${ }^{*} \mathrm{P}<0.05$.

(g) Representative microscopic image of mock treated and $\mathrm{H} 2 \mathrm{O} 2$ treated A549 cells detected by $\mathrm{Y}-\mathrm{H} 2 \mathrm{AX}$. (h) Representative quantitative data of $\mathrm{Y}-\mathrm{H} 2 \mathrm{AX}$ foci containing cells. Values are the mean $\pm \mathrm{SD}$ of three independent experiments. ${ }^{*} \mathrm{P}<0.05$. 